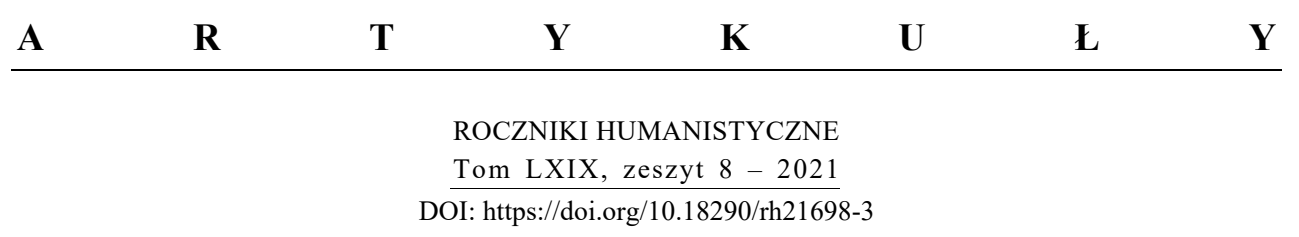

MARIA ISABEL GONZÁLEZ-REY

\title{
PHRASÉOLOGIE ET IDIOMATOLOGIE : DEUX TERMES CONCURRENTS OU COMPLÉMENTAIRES ?
}

\author{
PHRASEOLOGY AND IDIOMATOLOGY: \\ TWO COMPETING OR COMPLEMENTARY TERMS?
}

\begin{abstract}
Terminological discussions about the different elements that are dealt with in phraseology (Thun; Pilz; Gläser) have been abandoned in recent years. Yet the question is far from being resolved, not only as regards the naming of these elements, but also as regards their nature. Indeed, there continues to be a profusion of terms according to everyone's own theoretical frameworks (fixed expressions, phrasemes, phraseologisms, phraseological units, etc.). However, faced with this profusion of denominations and typologies within the discipline known as phraseology, the name of the field in question has had only one competitor since Bally proposed it: that of idiomatology (Guiraud), with its idiomatik variant in German (Burger et Jaksche), and its idiomatology translation into English (Makkai; Kavka and Zybert; Kavka "Compounding"). This observation prompts us to look for an explanation that goes beyond the mere random fate of words in their consolidation, by retracing the history of this rivalry, however short it may have been, in an attempt to understand the reasons for Bally's choice of terminology and how this choice came to prevail. We will do this by comparing the terms phraseology and idiotisms and their co-appearance in two 19th century works (Dupont, and Bochet et al.). This will certainly shed light on the division amongst specialists today, who are divided between a broad or narrow conception of the field, with more or less inclusive typologies. Above all, however, it will be an opportunity to reconcile phraseology and idiomatology as complementary fields..
\end{abstract}

Key words : phraseology; idiomatology; phraseological units; idiotisms; typologies.

MARIA IsABEL GonZÁLEZ-ReY - Professeure des Universités en Langue Française, Département de Philologie Classique, Française et Italienne, Faculté des Sciences Humaines (Campus de Lugo), Université de Santiago de Compostela (Espagne), Chef du Laboratoire FRASEONET (GI 2061) (www.phraseonet.com/es), e-mail: misabel.gonzalez.rey@usc.es; ORCID : https://orcid.org/00000002-8015-344X. 


\section{INTRODUCTION}

La terminologie d'une discipline joue un rôle essentiel dans sa consolidation et dans son prestige. En fait, c'est elle qui est chargée de lui donner entité et notoriété. Cependant, cette terminologie ne se crée pas du jour au lendemain ; elle a besoin de temps pour se stabiliser jusqu'à ce qu'elle devienne durable et d'usage commun à tous les spécialistes liés à ce nouveau champ du savoir. Pendant cette période de stabilisation, de nombreux termes concurrents prolifèrent, le temps que les plus utilisés finissent par s'imposer aux autres.

La phraséologie n'a pas échappé à cette contingence : plus d'un siècle après son émergence, ce domaine souffre encore d'une terminologie fluctuante, surdimensionnée et parfois contradictoire, soit pour désigner les différents éléments de son objet d'étude, soit pour désigner la propre nature de ces éléments, leurs sous-catégories ou encore leurs propriétés. Créé dans la seconde moitié $\mathrm{du} \mathrm{XX}^{\mathrm{e}}$ siècle, sous l'influence de Bally (vol. 1) et de ses adeptes russes, Vinogradov, entre autres -, ce domaine linguistique a connu une grande diffusion, d'abord à travers l'allemand (Burger et Jaksche; Thun ; Wotjak), ensuite à travers l'espagnol de Cuba (Carneado et Tristá), avant de gagner beaucoup d'autres pays dans le monde. Cette diffusion a amené une plus large connaissance du domaine mais aussi les premières discussions terminologiques, surtout dans le derniers tiers du $\mathrm{XX}^{\mathrm{e}}$ siècle $^{1}$. Cependant, elles ont été abandonnées depuis et c'est à peine si l'on ose y revenir aujourd'hui.

Pourtant, la question est loin d'être résolue. En effet, nous continuons à assister à une profusion des termes selon les cadres théoriques de tout un chacun (expressions figées, phrasèmes, phraséologismes, unités phraséologiques, etc.) et à une division des spécialistes selon une conception plus ou moins large du domaine, avec des typologies plus ou moins inclusives. Or, face à cette profusion de dénominations et de typologies à l'intérieur de la discipline, le nom du domaine (phraséologie) n'a eu qu'un seul concurrent depuis que Bally l'a proposé : celui d'idiomatologie (Guiraud), avec sa variante idiomatik en allemand (Burger et Jaksche), et sa traduction idiomatology en anglais (Makkai ; Kavka et Zybert ; Kavka, " Compounding »). Cela dit, l'acceptation unanime dont jouit aujourd'hui le mot phraséologie (et sa traduction littérale dans le reste des langues) nous donne à croire que cette concurrence n'a pas été trop forte et qu'elle n'a même pas laissé de traces. Mais, même si toutes les tentatives de remplacer le mot phraséologie ont

${ }^{1}$ Thun et Pilz ont été les premiers à exposer le «chaos terminologique » qu'il y avait en phraséologie. 
échoué, il nous faut aller à la recherche d'une explication qui aille au-delà du seul sort aléatoire des mots. C'est pourquoi nous nous proposons dans cette étude de retracer l'histoire de cette concurrence, toute courte qu'elle ait pu être, pour essayer de comprendre les raisons du choix terminologique de Bally et comment celui-ci est arrivé à s'imposer.

Pour cela, nous aborderons ici la problématique reliée à la concurrence des mots phraséologie et idiomatologie. Nous aurons l'occasion de voir que les raisons de cette rivalité en France se trouvent dans les aléas du mot phraséologie au fil des siècles, étroitement liés dès le départ à la didactique des langues, et dans la problématique posée par les idiotismes (ou gallicismes pour ce qui est du français) en regard de l'écart qu'ils présentent par rapport à la norme. Au cours de ce travail sur l'histoire du mot phraséologie, ou sur son étymologie -entendue comme "histoire des mots» et non seulement comme étude de leurs origines-, nous allons pouvoir constater que le terme naît sans lien avec l'idée que nous nous en faisons aujourd'hui, et encore moins avec la notion d'idiotismes. Le chemin que ce terme parcourra en coapparition avec celui d'idiotismes dans deux ouvrages du XIX ${ }^{\mathrm{e}}$ siècle, celui d'Hyppolyte-Auguste Dupont et celui de Bochet et al., va nous permettre de voir dans les mots phraséologie et idiomatologie deux termes non pas concurrents mais complémentaires.

\section{PRÉSENTATION DE LA PROBLÉMATIQUE}

La concurrence des termes phraséologie et idiomatologie pour nommer la discipline liée à l'étude des unités polylexicales a été objet de débat pendant quelques années dans des langues comme l'allemand ou l'anglais. Les arguments en faveur de l'un ou l'autre s'appuient notamment sur leurs origines étymologiques. Ainsi phraséologie provient du mot grec $\varphi \rho \alpha \sigma \varepsilon \circ \lambda \circ \gamma i \alpha$,

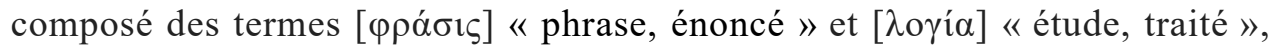

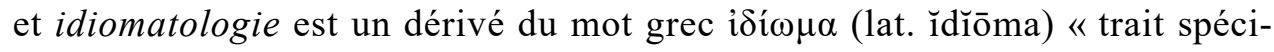
fique ; style particulier ». Il s'ensuit que le premier a un sens large, lié aux faits de la langue générale, alors que le second a un sens restreint, en lien avec tout ce qui se trouve à l'écart de cette langue générale. Dès le départ, leurs étymons les opposent, d'où le besoin de savoir d'où vient cette volonté de vouloir les rendre synonymes. 


\subsection{LE DÉBAT ENTRE LES MOTS PHRASÉOLOGIE ET IDIOMATOLOGIE}

Nous savons que le terme allemand phraseologie est arrivé en Allemagne traduit du russe, langue dans laquelle le mot avait été adopté, sans ambages, de Bally. Mais la préférence des auteurs germaniques penchait, déjà à l'époque, vers le mot Idiomatik, comme le montre le titre de l'œuvre de Burger et Jaksche : Idiomatik des Deutschen. Cela dit, le terme phraséologie s'est finalement imposé, à la vue du nouvel ouvrage de Burger et al., quelques années plus tard, Handbuch Der Phraseologie. Or deux œuvres de transition sont à citer entre ces deux dates, celles de Thun et de Pilz, respectivement. Dans le volume de Thun, nous trouvons tout un chapitre consacré à la question terminologique pour nommer la matière et ses éléments à partir de l'analyse des mots « idiomaidiotismus» (Probleme der Phraseologie 3-8) et "phrasis-phraseologia» (Probleme der Phraseologie 16-20), et cela dans plusieurs langues. L'étude de ces racines sur une même étendue de pages pourrait indiquer une position de non préférence de l'auteur pour l'un ou l'autre groupe de mots. Cependant, nous observons chez lui une certaine réticence envers le terme phraséologie dans l'affirmation suivante :

In keiner romanischen Sprache wird phraseologie usw. zur Bezeichnung der Wissenschaft von den phrases (faites) gebraucht. Auch in der engeren linguistischen Terminologie dieses Sprachen bezeichnet das Wort keine solche Disziplin. Selbst Bally, der von einigen Autoren als einer der Begründer « Phraseologie » angesehen wird, verwendet das Wort nur zur Bezeichnung der Gesamheit der «locutions », die eine Sprache besitzt ${ }^{2}$. (Probleme der Phraseologie 18)

Par contre, l'œuvre de Pilz traduit d'une manière explicite une forte conviction en faveur du mot phraséologie, à l'encontre de son concurrent germanique idiomatik. En effet, il est persuadé que l'adoption d'un terme reconnu internationalement ne peut que contribuer à la consolidation du domaine, comme l'ont fait les spécialistes russes avec le terme phraséologie, à l'opposé de ce que pensaient les spécialistes de l'Europe de l'Ouest. Cette position peut sembler d'autant plus surprenante que Pilz connaît bien les travaux de Makkai, qui avait déjà suggéré en 1975 la possibilité d'une sous-discipline, nommée idiomatology, placée à l'intérieur de la linguistique et chargée du

\footnotetext{
${ }^{2}$ Notre traduction: «Dans aucune langue romane [le mot] phraséologie n'est utilisé pour désigner la science des phrases [toutes] (faites). Le mot, pris dans la terminologie linguistique la plus étroite de cette langue, ne désigne pas non plus cette discipline. Même Bally, considéré par certains auteurs comme l'un des fondateurs de la 'phraséologie', n'utilise ce mot que pour désigner la totalité des 'locutions' que possède une langue ».
} 
langage idiomatique. Cela dit, l'affirmation de Gläser en 1984 introduit une nouvelle situation, à savoir l'acceptation de l'usage des deux termes en fonction d'une répartition géographique :

Another linguistic sub-discipline which has substantially contributed to the development of new terms is idiomatology. This is the British name of the linguistic discipline dealing with phraseological units [...]. Its equivalent in Continental linguistics, and in Soviet research in particular, is phraseology. (Terminological problems 348$)^{3}$

Dans cette citation, nous pouvons observer le constat des faits suivants : 1) l'origine anglaise donnée au mot idiomatology; 2) son caractère néologique ; 3) son statut de discipline linguistique ; 4) son objet d'étude (les unités phraséologiques) ; 5) sa valeur comme équivalent du mot phraseology pour référer à ce même objet d'étude ; et 6) la préférence de la linguistique continentale et de la recherche russe pour le terme phraseology.

Toutefois, les études de phraséologie réalisées en anglais britannique ou américain ont montré que le terme phraseology a prévalu aussi sur celui d'idiomatology. Or la bataille n'est pas encore gagnée car nous assistons au début du $\mathrm{XXI}^{\mathrm{e}}$ siècle à une nouvelle confrontation entre les mots phraseo$\log y$ et idiomatology en anglais. En effet, Kavka et Zybert, dans un travail de 2004, expriment leurs réserves par rapport au mot phraseology qui, pour eux, ne couvre pas «the vast domain of our interest since in common understanding it refers only to lexis $[\ldots]$ or that the very term 'phraseology' is derived from the base-term 'phrase', which for modern linguists has connotations of reference primarily to grammatical structures »(Glimpses 54-55). Ils préfèrent, par contre, celui d'idiomatology pour nommer la discipline qui s'occupe de l'ensemble des éléments préfabriqués du langage car : « [...] the term 'idiomatology' makes us regard the discipline as a truly linguistic one, treated as a field of science proper, i.e. one that has its objectives (goals) to probe and also its own methods of investigation » (Glimpses 55). Ils recourent à Grace (1981), créateur, pour eux, du mot idiomatology, en appui de leurs arguments :

It is appropriate to mention George W. Grace as he was the first not only to introduce the term 'idiomatology' but also to use it in the sense that coincides with our conviction that it shows principal features of a science. (Glimpses 55)

\footnotetext{
${ }^{3}$ Nous ne fournirons pas la traduction des citations en anglais, car il s'agit d'une langue internationale, largement connue dans le monde entier.
} 
Dans les citations de Kavka et Zybert, il y a une prise de positionnement évidente en fonction de critères conceptuels, ainsi qu'une volonté de faire pencher la balance en faveur du mot anglais ${ }^{4}$. Cela dit, d'autres phraséologues anglophones considèrent les deux mots comme des synonymes (Cf. Skandera ; Liontas), tandis que d'autres encore font prévaloir le mot phraseology comme le nom de la discipline qui s'occupe de tous les éléments préfabriqués du langage sur celui d'idiomatology, restreint aux idioms ou expressions idiomatiques, dites aussi imagées.

\subsection{L'IDIOMATOLOGIE ET LA THÉORIE DE L’ÉCART}

En fait, l'utilisation du mot idiomatologie comme terme synonyme, et même comme candidat à remplacer celui de phraséologie, relève d'une histoire où ni l'un ni l'autre n'a signifié au départ ce qu'il a signifié par la suite. En effet, nous allons voir que les emplois du mot phraséologie, dès sa première attestation à la Renaissance jusqu'au XVIII ${ }^{\mathrm{e}}$ siècle, rattachent le terme au système régulier de la langue, à travers la langue actualisée, c'est-à-dire les faits de langage, quels qu'ils soient. Par contre, celui d'idiomatologie fait son apparition en plein $\mathrm{XX}^{\mathrm{e}}$ siècle en France et va s'inscrire plutôt dans la théorie de l'écart, dans le sens que donne Dubois à ce mot dans son dictionnaire, à savoir : " On appelle écart tout acte de parole qui apparaît comme transgressant une de ces règles d'usage »(Dictionnaire 172) $)^{5}$. Dans ce sens, l'idiomatologie s'occupe de tout ce qui va, dans la langue, s'écarter de la norme linguistique.

En effet, le mot idiomatologie s'emploie en français d'abord dans le domaine de la littérature comparée pour référer à l'expression des particularités du langage. C'est dans ce sens que l'utilise Hatzfeld (1953) dans son œuvre sur la stylistique appliquée à la littérature romane pour désigner le domaine portant sur la langue courante comme style propre à une communauté nationale. Le terme passe ensuite dans le domaine de la recherche linguistique en phraséologie de la main de Guiraud qui désigne sous le nom d'idiomatologie tout ce qui a trait aux locutions, définies comme des expressions « d'origine marginale - le plus souvent technique, mais aussi dialectale, argotique ou affective, stylistique - qui sont passées dans la langue commune avec une valeur métaphorique et s'y sont conservées sous une forme figée et hors de l'usage

\footnotetext{
${ }^{4}$ Kavka avait déjà manifesté son appui en faveur de l'idiomatologie dans des travaux antérieurs (A Book on Idiomatology) et poursuivra cette ligne de recherche dans des travaux postérieurs, tels que « Compounding and Idiomatology ».

${ }^{5}$ Édition de 1973.
} 
normal »(Les locutions 7). En fait, Guiraud regroupe l'ensemble de ces locutions sous le nom d'idiomatologie pour en signaler les principales sources de formation : "Trois grandes sources alimentent l'idiomatologie : l'archaïsme, historique et externe d'une part, linguistique et interne l'autre; le style créateur d'images pittoresques et affectives ; le jeu des croisements synonymiques et homonymiques enfin $[\ldots] »$ (Les locutions 11).

Après Guiraud, très peu de travaux continuent d'employer le terme idiomatologie dans les études phraséologiques. Nous pouvons citer comme seul exemple ceux de Morgandinho, mais cette auteure l'utilise en tant que synonyme de phraséologie pour désigner aussi bien les locutions que les proverbes. Le mot idiomatologie, en définitive, n'a pas été retenu en phraséologie et il n'y eut même aucune controverse entre les deux mots en français, comme cela a été le cas, comme nous l'avons vu, pour l'allemand ou l'anglais. La question est de savoir pourquoi le mot ancien phraséologie l'a emporté sur le néologisme idiomatologie pour désigner le domaine qui lui est attribué de nos jours. Mais pour cela, nous devons porter toute notre attention sur le parcours parallèle et simultané des mots phraséologie et idiotismes dans le passé, en l'occurrence sur leur cohabitation dans deux ouvrages en particulier, celui de Dupont, consacré à l'enseignement du français langue maternelle, et celui de Bochet et al., dédié à l'enseignement du français langue étrangère. C'est à travers cette coapparition que nous pourrons bien connaître les rapports de force que ces deux termes ont toujours entretenus, beaucoup mieux que si nous les prenons séparément dans le temps.

\section{UN REGARD EN ARRIÈRE}

Les mots phraséologie et idiotismes sont attestés en français pour la première fois en $1678^{6}$ et $1558^{7}$, respectivement. Par rapport à ces attestations, le Trésor de la Langue Française informatisé (TLFi) définit le premier comme "recueil de phrases fait pour l'enseignement des langues", et le second comme « expression propre à une langue ». La différence entre ces deux définitions concerne notamment les notions de "phrase» et d' "expression propre ». Dans le premier cas, il s'agit de faits de langue, dans le sens large

\footnotetext{
${ }^{6} \mathrm{Cf}$. Le dictionnaire de Du Cloux, Vocabulaire françois avec une phraséologie convenable à tous ses mots, composé en faveur et pour l'usage de la jeunesse de Strasbourg. E.W. Schmuck, 446 p. Dans Quemada, Les Dictionnaires du français moderne (TFLi).

${ }^{7}$ Dans B. Des Périers, Nouvelles récréations et joyeux devis, éd. P. Jourda, II, p. 370 (TLFi).
} 


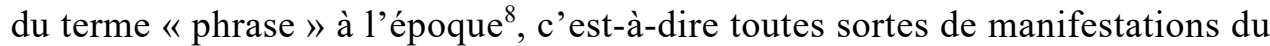
langage considérées comme régulières, destinées à l'apprentissage d'une langue. Dans le second, par contre, la notion n'embrasse qu'un groupe de manifestations considérées comme «propres » à une langue, un euphémisme qu'il faut traduire par «irrégulières » pour bien comprendre en quoi ces expressions sont propres, comme le montre la définition apportée par la suite dans la première édition du Dictionnaire de l'Académie française (DAF) (1694) :

s.m. Proprieté, maniere de parler, qui a quelque chose d'irregulier, mais qui est particuliere à une Langue. Cette particule mise de telle façon, cette construction, ce pleonasme est un idiotisme de la Langue Françoise, de la Langue Latine. chaque Langue a ses idiotismes. Il n'est en usage que parmy les gens de lettres?

Le français a donc ses propres idiotismes, nommés en l'occurrence gallicismes, un terme que le TLFi atteste pour la première fois en $1578^{10}$ et que le DAF définit ainsi dans sa première édition : « Maniere de parler particuliere à la langue françoise, \& contraire aux regles ordinaires de la grammaire. S'attaquer à quelqu'un, se battre avec quelqu'un, sont des gallicismes ».

Ce qui semble être bien accepté aux $\mathrm{XVI}^{\mathrm{e}}$ et $\mathrm{XVII}^{\mathrm{e}}$ siècles sur ce qu'il faut comprendre par phraséologie et idiotismes/gallicismes va, cependant, être remis en question au XVIII ${ }^{\mathrm{e}}$ siècle.

\subsection{DES IDIOTISMES ET DES GALLICISMES}

La bataille qui va se livrer en plein XVIII ${ }^{\mathrm{e}}$ siècle pour la défense de la langue française va marquer par la suite toute la différence entre ce qui est phraséologique et ce qui est idiomatique. En effet, à l'époque, l'opinion générale considère les gallicismes comme une espèce de barbarismes. À l'encontre de cette opinion, Beauzée pose que «l'essence du gallicisme consiste en effet à être un écart de langage exclusivement propre à la langue françoise [mais qui] en françois est à sa place, \& il y est ordinairement pour éviter un vice » (gallicisme 450). Depuis cette défense faite dans les articles dédiés aux termes gallicisme et idiotisme dans l'Encyclopédie de Diderot et d'Alembert (Beauzée 450 et Beauzée $497^{11}$ ), elles ont «droit de cité dans la langue » (TLFi).

${ }^{8}$ Le seul qu'ait le mot jusqu'au XVIII" siècle, à savoir celui de «tout assemblage de mots »

${ }^{9}$ Nous reproduisons telles quelles l'orthographe et la ponctuation utilisées dans cette première édition du DAF.

${ }^{10}$ Dans H. Estienne, Deux dialogues du nouveau langage françois italianizé, éd. P. Ristelhuber, 1578, t. 2, p. 177 (TLFi).

${ }^{11}$ Éditions de 1757 et de 1766, respectivement. 
(Bárdosi 75). Leur présence n'est donc pas surprenante dans les grammaires françaises destinées à l'enseignement du français langue maternelle pendant les $\mathrm{XVIII}^{\mathrm{e}}$ et $\mathrm{XIX}^{\mathrm{e}}$ siècles. Toutefois, cette présence est extrêmement variée, signalée sous la dénomination d'idiotismes, de gallicismes et parfois même de barbarismes, lorsque l'auteur décide de les considérer comme tels. Il en va autrement, cependant, dans les ouvrages qui portent le mot de phraséologie sur la couverture et avec lequel ceux-là devront coapparaître.

\subsection{DES GALLICISMES DANS LA PHRASEOLOGIE}

Le mot phraséologie apparaît pour la première fois sur la couverture d'une grammaire destinée à l'enseignement du français comme langue maternelle dans l'ouvrage de Dupont: Phraséologie française élémentaire ou Nouveaux exercices de grammaire. Ce volume représente la deuxième partie du livre Méthode pour mettre la Grammaire à la portée de l'enfance et constitue, avec la première partie, un cours complet pour l'enseignement de la langue française aux petits Français. Son auteur situe cette deuxième partie dans la catégorie des cours pratiques pour la maîtrise de la langue normée, en complément à la première, plus théorique, qui traite des notions relatives à la grammaire, en général, et aux parties du discours, en particulier ${ }^{12}$. Dans cet ouvrage le terme phraséologie renvoie à plusieurs réalités dans l'enseignement du français comme langue maternelle :

- La phraséologie y est considérée dans un sens pratique, en association avec l'entrainement à la langue « française » au moyen d' « exercices »;

- Elle est représentative des règles ordinaires de la langue et, comme partie pratique, elle est faite pour s'entrainer à la maîtrise de la " grammaire »;

- La phraséologie y est présentée sous la forme d'une méthode nouvelle d'enseignement (grâce au terme « nouveaux exercices »), valable dès un niveau précoce d'apprentissage ( élémentaire »).

- Cette méthode consiste en une démarche particulière qui met le raisonnement de la langue à la portée des élèves au moyen de phrases, que l'auteur nomme faits et qu'il a conçues pour illustrer les règles grammaticales.

En bref, la phraséologie est prise ici aussi dans un sens large, assimilée à toutes sortes de faits de langue employés dans un but didactique. Toutefois, ces faits correspondent aux manifestations actualisées des normes de grammaire. Or cette phraséologie, que nous pouvons qualifier de "générale »- les faits illustrant les règles ordinaires de la langue dans son entièreté -, contient aussi une phraséologie "particulière " dans l'œuvre de Dupont (chapitre

\footnotetext{
${ }^{12}$ Pour plus de détails sur l'analyse de cet ouvrage, voir González-Rey « La phraséologie ».
} 
«Étude de la phrase » 74-76), fondée sur les idiotismes (gallicismes) qui, eux, contreviennent ces règles en question. Il s'agit, dans ce cas, d'une phraséologie prise au sens étroit située à l'intérieur d'une phraséologie prise au sens large. Cette double considération de la phraséologie, présente chez Dupont le seul d'ailleurs à la contenir, à notre connaissance -, va cependant connaître un effet contraire dans le cas de l'enseignement des langues étrangères.

\subsection{DES IDIOTISMES OU DE LA NOUVELLE PHRASÉOLOGIE}

Le mot phraséologie va commencer à perdre son sens large et à être assimilé au sens étroit que va lui imposer les idiotismes sous l'effet de l'enseignement des langues étrangères, notamment dans des méthodes d'apprentissage $\mathrm{du}$ français publiées à l'étranger. Ainsi l'ouvrage de Bochet et al. illustre parfaitement cette nouvelle voie de convergence entre deux termes qui, même si l'un incluait l'autre, se distinguaient jusqu'ici par la nature régulière ou irrégulière de leurs éléments. En effet, cet ouvrage présente deux titres très significatifs : le premier Cours pratique de Langue Française. Phraséologie et le second, Recueil d'Idiotismes ou Nouvelle phraséologie française et italienne par P. ${ }^{\text {re }} \mathrm{A}$. Bochet (précédée de celles de MM. Moretti, Chev. Cardinelli et J. Ph. Barberi, et suivie des Entretiens mémorables de Socrates). Dans le premier titre, le mot phraséologie s'accorde bien avec son sens connu jusqu'alors comme phrases modèles pour les cours pratiques dans l'enseignement d'une langue normée; dans le second, le caractère de "nouvelle » est attribué à une phraséologie synonyme d'idiotismes.

Dans sa structure, l'ouvrage contient 4 chapitres portant sur une phraséologie bilingue français-italien, relevée par chaque auteur selon ses propres critères. Le premier chapitre comprend la phraséologie recueillie par M. Moretti et se divise en deux parties, la première portant le titre de «Phrases italiennes dont les mots, et non le sens, ressemblent aux mots français » et attirant l'attention sur les faux-amis dans les deux langues, et la seconde, le titre de "Italianismes ou idiotismes », avec des phrases ou des locutions ayant des structures et du lexique tout à fait différents dans les deux langues. Le deuxième chapitre correspond à la phraséologie rassemblée par le Chevalier Cardinelli, composé aussi de deux parties, la première intitulée «Phrases françaises et italiennes » et offrant des phrases où l'un des termes peut être polysémique en français, ce qui rend la plupart du temps des équivalents tout à fait différents en italien, et la seconde nommée «Phrases élémentaires», avec des énoncés complets en français et des équivalents absolus ou partiels en italien. Dans le troisième chapitre, consacré à la phraséologie de J. Ph. Barberi 
et intitulé "Phraséologie familière », l'auteur incorpore un mélange de proverbes, de phrases et de locutions sans suivre d'ordre déterminé ni de registre concret, accompagnés de « la vraie correspondance entre la manière de dire en français et la manière de dire en italien » (Cours pratique 10). Finalement, le dernier chapitre, de P. ${ }^{\text {re }}$ Alexis Bochet, nommé « Nouvelle phraséologie », sert de titre à tout l'ouvrage et semble annoncer une avancée par rapport à la phraséologie recueillie dans les chapitres précédents. En fait, il s'agit d'une liste de phrases, elliptiques ou métaphoriques de la langue italienne, sous forme d'énoncés complets, tirées de l'ouvrage d'Antonio Lissoni Fraseologia italiana o Raccolta di ventimila frasi o modi di dire, per ordine di alfabeto, traduites en français. L'ordre suivi en italien porte sur l'un des formatifs substantifs mis en relief dans l'énoncé, pouvant constituer pour un apprenant italien de français langue étrangère une difficulté de traduction.

Ce qui rassemble donc ces quatre chapitres est la perspective interlinguistique adoptée entre la langue d'apprentissage et la langue maternelle. Le volume en soi constitue un véritable manuel de traduction centré essentiellement sur les difficultés de la version, de la langue étrangère à la langue maternelle. C'est donc essentiellement à travers les divergences que se présente cette nouvelle phraséologie, comme indiqué par l'éditeur de l'ouvrage dans le «Simple Avis » (Cours pratique 10). Bien que dans le livre de Bochet et $a l$. cette phraséologie continue de se référer à la partie pratique d'un cours de langue à travers des phrases choisies comme modèle d'enseignement, elle est assimilée aux idiotismes de cette langue d'apprentissage en ce que ceuxci constituent des écarts non pas par rapport au système intralinguistique de la langue étrangère mais par rapport à celui de la langue maternelle de l'apprenant. Or, bien que ce soient les idiotismes qui exercent ici leur influence sur le changement sémantique du mot, le terme phraséologie s'impose encore dans tout le volume.

Curieusement, ce sera là probablement le dernier moment où les deux termes se côtoieront. En effet, le mot phraséologie va perdre définitivement son sens large originel et tomber dans un discrédit qui avait déjà commencé dans son usage social et même littéraire vers la fin du XVIII ${ }^{\mathrm{e}}$ siècle $^{13}$. Dans l'optique de l'usage de la langue, la répétition de phrases toutes faites, toutes correctes qu'elles puissent être, lasse, a fortiori provenant de personnes lettrées qui doivent parler en public et qui sont censées être originales dans leur mode d'expression, écrite ou orale. Or, si l'on ajoute à cela le fait qu'à

${ }^{13}$ Cf. Le Babillard, 10 avril - I, no XX, p. 321 - dans Proschwitz Beaumarchais, p. 282: Qu'un misérable Rhéteur... revêtisse d'une vaine et sonore phraséologie les Plagiats mutilés (TLFi). 
l'époque, et pendant un siècle de plus, le degré d'analphabétisme en France est assez élevé, le passage d'un enseignement de la langue fondé sur une phraséologie entendue comme ensemble de phrases correctes, servant de modèles à la production langagière dans l'apprentissage d'une langue, à un enseignement associé aux phrases « reproduites » et irrégulières, - surtout du point de vue de leur traduction dans la langue maternelle des apprenants -, et signalés la plupart par les auteurs français de grammaires françaises au XIX ${ }^{\mathrm{e}}$ siècle, se comprend aisément. Le mot phraséologie ne connaîtra un regain de vitalité qu'à partir de 1909, avec Bally qui l'adopte sans ambages en linguistique. La question, dès lors, est de savoir pourquoi.

\section{CONCURRENCE ET COMPLÉMENTARITÉ}

Le bref panorama exposé ci-dessus met en évidence un constat : celui d'un contraste entre l'existence du mot phraséologie en tant que nom générique pour désigner une classe, et celle du mot idiotisme, en tant que mot spécifique pour désigner des éléments particuliers. La concurrence entre ces deux termes, tracée tout au long de leur étymologie, nous permet de constater que la lutte entre les deux n'en finit pas pour autant avec Bally. En effet, chacun des deux va chercher à développer son pendant dans la catégorie contraire : les idiotismes chercheront à s'octroyer un nom générique pour les désigner comme classe de mots et la phraséologie cherchera un nom déterminé pour désigner l'ensemble des éléments spécifiques qui se poseront comme objet d'étude. Il résulte pour les premiers la tentative du néologisme idiomatologie, dont nous avons vu le sort plus haut, et pour le second, toute une myriade de noms selon les différentes typologies présentées par les spécialistes du domaine. Or le succès d'un mot auprès de ses usagers demeure un mystère. On serait tenté de croire qu'il est plus facile d'adopter un mot nouveau que de donner un nouveau sens à un mot déjà créé pour nommer une nouvelle réalité. Il n'en est pourtant rien, comme nous allons le voir.

\subsection{LES INCONVÉNIENTS DES NÉOLOGISMES}

Gläser (« Terminological problems »), dans son étude sur les problèmes terminologiques en linguistique, signale les difficultés que posent les néologismes dans le domaine phraséologique en tant que termes concurrents de termes anciens et préétablis, et qui, dans une même langue, peuvent se chevaucher, et, dans d'autres, peuvent ne pas toujours renvoyer à la même définition 
du concept auquel ils se réfèrent. Ceci nuit non seulement à la délimitation de l'objet d'étude et à sa dénomination, mais aussi à l'internationalisation des termes et à leur collecte dans les dictionnaires bilingues ou multilingues de linguistique. Ainsi, elle signale, en exemple, le mot idiom, mot générique employé en anglais pour désigner tous types de groupes de mots préétablis dans cette langue, alors que ce terme dans d'autres langues, telles que le russe ou l'allemand, est lié à la notion d'idiomaticité et ne désigne qu'un seul type d'éléments phraséologiques, celui « which cannot be derived from the meanings of its constituents, but is often obscured or fossilized» («Terminological problems » 349). À l'opposé, elle signale la profusion de synonymes qui peuvent surgir dans une même langue pour signaler les groupes de mots préfabriqués, rivalisant entre eux pour se rapporter au même objet, d'où aussi le problème consécutif à leur traduction dans d'autres langues dû à un manque de coordination terminologique entre les spécialistes, qui préfèrent offrir leur contribution personnelle à la terminologie phraséologique : " This range of synonymous terms is by no means an extreme case, but quite a natural phenomenon where absence of terminological coordination allows for new individual coinages which are often presented as novel research » (« Terminological problems »349).

En français aussi, le phénomène est naturel puisque nous nous retrouvons, encore aujourd'hui, face à une prolifération de termes concurrents pour nommer globalement l'objet d'étude de la phraséologie, tels que unités phraséologiques (Gréciano), phrases figées (Gross, "Les limites »), phrasèmes (Mel'čuk, "Phrasemes »), expressions figées (Gross, Les expressions), phraséologismes (Kauffer), segments répétés (Legallois), entre beaucoup d'autres. Toutes ces différentes dénominations posent, en effet, un problème de coordination terminologique entre spécialistes d'une même langue d'étude et de travail, et aussi un problème d'équivalences pour l'établissement d'une terminologie internationale universelle. Les raisons de cette diversité peuvent être reliées aux diverses approches théoriques où chaque spécialiste encadre ses études, mais aussi à la priorité donnée à ce qui caractérise le mieux, à leurs yeux, ces éléments : le figement, la polylexicalité, la répétition, le caractère unitaire, etc. Or cette façon de nommer l'objet d'étude en fonction des cadres théoriques ou des caractéristiques priorisées de ces groupements de mots se retrouve aussi au niveau des différentes dénominations que propose chaque spécialiste pour chaque sous-type selon sa typologie. Ainsi peut-on trouver des termes aussi variés que formules routinières (Coulmas), énoncés liés (Fónagy), pragmatèmes (Mel'čuk), phraséologismes pragmatiques (Kauffer), etc. pour 
se référer aux éléments préétablis du langage en lien avec des situations d'énonciation déterminée.

Or le problème terminologique relevé ici pour nommer non seulement l'objet d'étude de la phraséologie en français, mais aussi les différents soustypes qui le forment, ne se pose pas pour désigner la discipline. En effet, le terme phraséologie proposé par Bally en 1909 s'est imposé sans réserve, et cela malgré la concurrence du néologisme idiomatologie proposé par Guiraud. Bien que nous ne puissions pas expliquer les raisons de la réussite de ce mot entre les spécialistes, nous pouvons essayer de comprendre quelles ont été les raisons de ce choix de la part de Bally.

\subsection{LES ATOUTS DES MOTS ANCIENS}

Si nous nous tournons un instant vers le mot stylistique, rappelons-nous ce que Bally nous confie à ce propos dans son Traité de Stylistique française I : "C'est peut-être une faiblesse que d'avoir reculé devant la création d'un terme nouveau » (Traité I IX). Dans cet ouvrage, on voit bien, en effet, comment l'auteur s'évertue à attribuer à ce mot un sens nouveau par rapport à celui qu'il avait alors :

Je sais qu'on a attribué à ce mot [stylistique] des significations fort diverses [...] à mon sens aucune des définitions proposées jusqu'ici de la stylistique n'est valable [...] notre science [...] ne se confond ni avec l'art d'écrire, ni avec la rhétorique, ni avec la littérature, ni avec l'histoire de la langue; mais elle doit constamment s'occuper de ces disciplines pour les empêcher d'envahir son domaine propre.[...] Je sais que de la sorte on risque d'enfoncer des portes ouvertes; mais si la critique reconnaît quelque originalité à mon effort, elle la trouvera dans le caractère strictement expérimental de la recherche [...]. (Traité I IX-X)

Or, en ce qui concerne le mot phraséologie, Bally a dû certainement agir de la même façon : il a pu reculer "devant la création d'un terme nouveau ». Il a pris un mot disponible dans la langue et lui a donné un nouveau sens, un sens adapté à sa notion de stylistique. Or la stylistique, telle que conçue par Bally, est définie comme l'étude des moyens expressifs de «la langue d'aujourd'hui, dans ses manifestations les plus vivantes, les plus voisines de la pensée spontanée » (Traité I IX). Cette stylistique a donc pour vocation d'embrasser la langue entière, comme le faisait la phraséologie à ses débuts. Les faits d'expression viennent alors remplacer les faits de langues. Mais dans cette substitution, Bally récupère le mot phraséologie et le déplace vers un espace plus restreint pour se référer aux faits d'expression pourvus d'une 
cohésion sémantique soit partielle soit absolue et qu'il nomme groupes phraséologiques. Ce sont des groupes de mots qui forment des unités de sens, ou à rebours, ce sont des éléments dont «l'unité psychologique excède les limites de l'unité graphique et s'étend sur plusieurs mots» (Traité I 65). C'est l'ensemble de ces faits qu'il regroupe «sous le terme général de phraséologie » (Traité I 66).

D'un point de vue sémantique, cette conception attribue à la phraséologie un sens restreint puisque ses éléments, identifiables à la cohésion sémantique de leurs composants, s'opposent au reste des éléments de la stylistique, situés dans la combinatoire libre de la langue. Cependant, d'un point de vue grammatical, bien que dans la typologie de Bally seuls les syntagmes (ou locutions) semblent rendre compte des éventuels groupements phraséologiques qui forment les séries et les unités phraséologiques, - ce qui exclut, apparemment, les phrases en tant qu'énoncés complets -, il existe aussi une phraséologie exclamative faite de mots (Allons!, Tiens!) et de phrases (C'est bien fait!, Que voulez-vous ?, Qui sait?). De ce fait, le sens que Bally donne au mot phraséologie pour désigner l'ensemble de la combinatoire fixe d'une langue est large du point de vue grammatical, bien qu'il soit étroit par rapport à la stylistique, puisqu'il s'agit d'une phraséologie qui ne couvre qu'une partie de la langue.

Cela dit, la phraséologie de Bally contient à son tour une partie qui se distingue du reste des groupements phraséologiques, une partie composée de gallicismes. Il y regroupe aussi bien des expressions imagées, telles que mettre la puce à l'oreille, risquer sa peau, que des expressions propres à la conversation, telles que À la bonne heure! ou Je n'en peux mais. Les premières «contiennent des images (affectives ), c. à d. des images perçues à la fois confusément et fortement " (Bally, Traité II 157) et les secondes sont impossibles « de prononcer sans une intonation exclamative» (Bally, Traité I 166). Pour Bally les gallicismes se trouvent au cœur de la langue parlée, en tant que produits de la spontanéité des locuteurs et fruits de l'expression de leurs sentiments. Ces expressions, dont les images sont « empruntées au monde sensible le plus voisin des sujets parlants »(Bally, Traité I 291-292), sont le plus souvent employées dans la langue de la conversation et présentent un contraste " frappant " avec leur signifié. La manière de les identifier consiste à en chercher l'équivalence dans une construction plus régulière, comme il l'indique pour les exemples suivants : À la bonne heure! reviendrait à dire « Je suis satisfait », ou bien Prendre ses jambes à son cou, "s'enfuir précipitamment » 
(Traité I 166). Ici encore, les gallicismes sont présentés comme des constructions spécifiques, à l'écart de la langue régulière.

On le voit bien : Bally conçoit ainsi une phraséologie binaire, telle qu'elle existait auparavant, et de ce fait, nous nous retrouvons face au même problème que le mot avait, avant lui, dans le système général de la langue, lorsqu'il s'opposait aux idiotismes : un sens large qui englobe aussi bien des mots simples que des syntagmes (où seraient inclus les locutions, les collocations et même les mots composés ${ }^{14}$ ) ou encore des phrases ; ou un sens étroit, limité aux idiotismes proprement dits.

Il est donc dans le sort du mot phraséologie que de se réserver un sens large, hérité de son passé, afin d'embrasser le plus grand nombre de constructions possible, quels que soient les critères adoptés par les différents auteurs. À l'opposé, se trouvent les idiotismes dont le sort a toujours été de s'écarter de la norme et de se distinguer grâce à cela. Or il n'a pas été toujours facile à la phraséologie de les englober sans courir le risque d'être confondue avec eux ou de n'être limitée qu'à leur seule présence. D'où le positionnement de phraséologues qui prennent la phraséologie au sens large et celui de ceux qui la prennent au sens étroit.

\subsection{UN RAPPORT DE COMPLÉMENTARITÉ}

Cela dit, même si l'histoire des mots phraséologie et idiomatologie nous a fait voir les rapports de force qu'ils ont toujours entretenus, et même si les idiotismes n'ont pas réussi à imposer le terme générique qui aurait pu leur correspondre, celui d'idiomatologie, pour désigner au moins un sous-domaine à l'intérieur de la phraséologie, il nous semble convenable de postuler cette dénomination comme complémentaire de celle de phraséologie.

En effet, la présence du terme idiomatologie dans les langues a montré qu'il y a une certaine conscience d'un quelque chose qui les distingue et qui rend chacune particulière à sa façon. Bien que le terme n'apparaisse pas dans les dictionnaires d'usage du français, sa présence est transversale, on l'a vu, dans plusieurs domaines (dialectologie, littérature, linguistique, anthropologie), en lien avec ce qui a trait, à la fois, à la spécificité de leurs objets d'étude et à leur divergence par rapport à d'autres éléments. En linguistique, le mot

${ }^{14} \mathrm{Cf}$. Śliwa (Unités polylexicales 408 ) pour qui il existerait, dans le cadre des syntagmes nominaux, trois catégories possibles : " les expressions idiomatiques nominales, les collocations adjectivo-nominales et les noms composés ». Cette auteure se sert des notions d'endocentrisme et d'exocentrisme pour définir les noms composés et distinguer les noms composés exocentriques des locutions et les noms composés endocentriques des collocations. 
contient donc deux perspectives : l'une, intrinsèque, tournée vers les particularités d'une langue donnée, dans toutes ses manifestations (écrites, orales ou culturelles), des particularités qui se révèlent à travers la comparaison avec le reste des faits de langage ; l'autre, extrinsèque, tournée vers les différences entre tous les faits d'une langue (réguliers ou irréguliers) et ceux d'une autre langue. Dans les deux cas, c'est non seulement la théorie de l'écart qui caractérise le mot, mais aussi l'approche comparée. Ainsi, dans la perspective intrinsèque, c'est à travers la comparaison entre la norme et ce qui s'en écarte que se manifestent les particularités d'une langue, et de ce fait, son idiomaticité, et dans la perspective extrinsèque, c'est la comparaison entre les constructions générales et les particularités de chaque langue que se manifeste l'écart entre l'idiomaticité de chacune d'entre elles. Or le passage d'une perspective à l'autre s'est produit dans les deux ouvrages analysés plus haut: les idiotismes présentés dans le livre de Dupont ont montré une idiomatologie intrinsèque à l'intérieur d'une phraséologie générale, tandis que ceux du livre de Bochet et al. ont évolué vers une idiomatologie extrinsèque à travers une phraséologie contrastive entre deux langues. Ceci nous permet de soutenir que si, à l'heure actuelle, la phraséologie se situe dans l'intralinguistique, en lien avec les éléments préfabriqués d'une langue donnée, l'idiomatologie, quant à elle, peut se situer dans l'interlinguistique ${ }^{15}$. En fait, c'est bien en tant qu'étude comparée des langues que Hatzfeld (1944-1946) définit le mot :

El verdadero método de la idiomatología parece estar destinado a ser comparativo. $[\ldots]$ el estudio comparado de las lenguas romances [...] podría alcanzarse por medio de la idiomatología comparada. Este fecundo campo lingüístico literario incluye necesariamente la estética del lenguaje, la psicología de las naciones y la historia de las civilizaciones. (Nuevas investigaciones estilísticas 72$)^{16}$

Dans cette définition large du terme, le mot idiomatologie trouve sa place et sa fonction par rapport à la phraséologie. En effet, il se réconcilie avec tout ce qui l'opposait à elle en se présentant comme complémentaire. Les deux termes n'ont plus à rivaliser pour occuper le même espace. Au contraire, chacun joue dans un camp différent, mais rattaché l'un à l'autre.

\footnotetext{
${ }^{15}$ Pour plus de détails sur une approche idiomatologique en phraséotraductologie littéraire, voir González-Rey (sous presse).

${ }^{16}$ Notre traduction: «La véritable méthode de l'idiomatologie semble destinée à être comparative. [...] l'étude comparée des langues romanes [...] pourrait être réalisée au moyen de l'idiomatologie comparée. Ce champ fertile linguistique littéraire comprend nécessairement l'esthétique du langage, la psychologie des nations et l'histoire des civilisations ».
} 


\section{CONCLUSIONS}

Le mot phraséologie proposé par Bally pour nommer l'ensemble des expressions à cohésion relative ou absolue dans une langue existait bien avant qu'il ne décide de l'employer, mais dans un sens bien différent de celui que nous lui connaissons aujourd'hui. Les premières attestations du terme en français montrent le sens large dans lequel il était utilisé et sa fonction didactique, de nature pratique, dans l'enseignement des langues. Or ce sens va s'affirmer d'autant plus dans la tradition française qu'il sera réservé aux faits de langue réguliers, auxquels s'opposeront les idiotismes ou, en l'occurrence, les gallicismes, considérés comme des constructions propres de la langue, mais irrégulières.

La coapparition des deux termes, phraséologie et idiotismes, dans deux ouvrages du XIX ${ }^{\mathrm{e}}$ siècle a montré les rapports qui les liaient. Dans l'œuvre de Dupont, le sens large et normé donné à la phraséologie a marqué une position intralinguistique dominante par rapport aux idiotismes, considérés comme des irrégularités qu'il fallait tolérer. Par contre, dans l'œuvre de Bochet et al., grâce à la perspective interlinguistique de la langue à apprendre en regard de la langue maternelle, les idiotismes ont réussi à s'imposer au point d'en modifier la donne et de transformer le mot phraséologie en nouvelle, forçant ainsi le terme à les tenir en compte en premier lieu dans l'enseignement de la langue, en l'occurrence, la langue étrangère. Or cela a été possible grâce à un procédé particulier, la traduction, de telle sorte que s'il fallait faire un partage entre les mots phraséologie et idiomatologie, il faudrait attribuer au premier l'étude intralinguistique des faits langagiers dans une langue donnée, et au second l'étude interlinguistique des faits de la langue source et de la langue cible au moyen de la traduction.

En effet, le mot idiomatologie a tenté sa chance pour concurrencer celui de phraséologie dans le but soit de désigner ce qu'étaient les idiotismes proprement dits (Guiraud), soit d'embrasser tous les éléments considérés comme phraséologiques. Or le terme phraséologie a redoré son blason grâce à Bally, après être tombé en discrédit et a fini par s'imposer. Mais il continue à diviser les spécialistes sur la délimitation du domaine qu'il désigne et sur la nature de son objet d'étude. Nous en sommes donc au même point qu'aux siècles précédents où la phraséologie avait un sens large et les idiotismes un sens étroit. De son côté, le mot idiotisme, depuis sa désannexion d'une phraséologie longtemps considérée comme générale et régulière et qui l'incluait comme une 
partie en marge de la langue, a été en quête d'un nom dérivé d'un étymon qui pouvait le représentait et lui donner ses lettres de noblesse, mais en vain.

Aujourd'hui, ils peuvent ne plus être concurrents pour une même discipline, mais complémentaires en ceci que la phraséologie s'occupe de l'étude des faits d'expression, dans le sens de Bally, tandis que l'idiomatologie s'érige en une approche particulière pour les traduire dans une autre langue (thème ou version) comme garant d'une équivalence idiomatique entre langue source et langue cible.

\section{RÉFÉRENCES BIBLIOGRAPHIQUES}

Bally, Charles. Traité de Stylistique française. Vol. I. Carl Winter's Universitätsbuchhandlung, 1921 [1909].

Bally, Charles. Traité de Stylistique française. Vol. II. Librairie Georg \& Cie-Klincksieck, 1951 [1909].

Bárdosi, Vilmos. «Contribution à l'histoire de la phraséologie française des origines jusqu'à Michel Bréal ». Acta Universitatis Szegedinensis, Acta Romanica, t. XIV, Studia Lexicographica Neolatina II, 1990, pp. 61-132.

Beauzée, Nicolas (de). « Gallicisme ». Encyclopédie ou Dictionnaire raisonné des sciences, des arts et des métiers. Livre VII, publié par Jean-Baptiste le Rond D'Alembert et Denis Diderot, Paris : Briasson, David, Le Breton, Durand, 1757, p. 450.

Beauzée, Nicolas (de). «Idiotisme». Encyclopédie ou Dictionnaire raisonné des sciences, des arts et des métiers. Vol. VIII, publié par Jean-Baptiste le Rond D'Alembert y Denis Diderot. Paris : Briasson, David l'aîné, Le Breton, Durand, 1766, p. 497.

Bochet, Moretti et al. Cours pratique de Langue Française. Phraséologie. Recueil d'Idiotismes ou Nouvelle phraséologie française et italienne par $\mathrm{P} .{ }^{\mathrm{re}} \mathrm{A}$. Bochet (précédée de celles de MM. Moretti, Chev. Cardinelli et J. Ph. Barberi, et suivie des Entretiens mémorables de Socrates). Venise : Pierre Naratovich, 1846.

Burger, Harald et Harald Jaksche. Idiomatik des Deutschen. Niemeyer Verlag, 1973.

Burger, Harald, Annelies Buhofer et Ambros Sialm. Handbuch Der Phraseologie. De Gruyter, 1982.

Carneado Moré, Zoila et Antonia María Tristá Pérez. Estudios de Fraseología. Academia de Ciencias de Cuba, 1985.

Coulmas, Florian. «On the Sociolinguistic Relevance of Routine Formulae ». Journal of Pragmatics, vol. 3, nº 3-4, 1979, pp. 239-266.

Dictionnaire de l'Académie française en ligne. www.dictionnaire-academie.fr/.

Dubois, Jean, Mathée Giacomo, Louis Guespin, Christiane Marcellesi, Jean-Baptiste Marcellesi, Jean-Pierre Mevel. Dictionnaire de linguistique et des sciences du langage. Larousse, 1973.

Dupont, Hippolyte-Auguste. La Grammaire à la portée de l'enfance. 2e partie : Phraséologie française élémentaire ou Nouveaux exercices de grammaire ( $5^{\mathrm{e}}$ édition). Paris: Librairie élémentaire de E. Ducrocq et Librairie de Langlois et Leclercq, 1856 [1833]. 
Estienne, Henri. Deux dialogues du nouveau langage françois italianizé, introduction et notes de Paul Ristelhuber, vol. 2. Geneva: Alphonse Lemerre, 1578. gallica.bnf.fr/ark:/12148/bpt6k 411445t.texteImage\# (édition de 1885). Consulté le 01 octobre 2021.

Fónagy, Ivan. Situation et signification. John Benjamins, 1982.

Gläser, Rosemarie. «Terminological problems in linguistics, with special reference to neologisms ». LEXeter '83 proceedings : papers from the International Conference on Lexicography at Exeter, 9-12 September 1983, édité par Reinhard R. K. Hartmann, Max Niemeyer Verlag, 1984, pp. 345-351.

González-Rey, M ${ }^{\mathrm{a}}$ Isabel. (sous presse). « Origines pédagogiques de la phraséologie : De la didactique des langues à la didactique de la traduction ».

González-Rey, M ${ }^{\mathrm{a}}$ Isabel. «Idiomaticidad e idiomatización en traducción literaria ». Clina, 6/2, numéro spécial Phraseology in Translation and Interpreting in the $21^{\text {st }}$ Century / Fraseología en la traducción e interpretación en el s. XXI, 2021, pp. 33-50. DOI 10.14201/clina2020623350.

González-Rey, M ${ }^{\mathrm{a}}$ Isabel. «La phraséologie dans l'étude du français langue maternelle : des faits de langue d'Hippolyte-Auguste Dupont aux faits d'expression de Charles Bally ». Yearbook of Phraseology, $\mathrm{n}^{\mathrm{o}}$ 11, 2020, pp. 171-197. DOI 10.1515/phras-2020-0009.

Grace, George W. Essay on language. Hornbeam Press, 1981.

Gréciano, Gertrud. «Pour un apprentissage des unités phraséologiques », Nouveaux cahiers d'Allemand, $\mathrm{n}^{\circ}$ 2, 1984, pp. 95-113.

Gross, Gaston. Les expressions figées en français. Noms composés et autres locutions. Ophrys, 1996.

Gross, Maurice. «Les limites de la phrase figée ». Langages, $\mathrm{n}^{\circ}$ 90, 1988, pp. 7-22.

Guiraud, Pierre. Les locutions françaises. Presses universitaires de France, 1980 [1961].

Hatzfeld, Helmut Anthony. « Nuevas investigaciones estilísticas en las literaturas románicas (1932-1945) ». Boletín de Filología de la Universidad de Chile nº4, 1944-1946, pp. 7-77.

Hatzfeld, Helmut Anthony. A Critical Bibliography of the New Stylistics Applied to the Romance Literature, 1900-1952. Université de Caroline du Nord, 1953.

Kauffer, Maurice. «Le figement des 'actes de langage stéréotypés' en français et en allemand». Pratiques, $\mathrm{n}^{\mathrm{0}}$ 159/160, 2013, pp. 42-54.

Kavka, Stanislav. " Compounding and Idiomatology ». The Oxford Book of Compounding, édité par Rochelle Lieber et Pavol Štekauer. Oxford University Press, 2009.

Kavka, Stanislav. A Book on Idiomatology. Université de Žilina, 2003.

Kavka, Stanislav; Jerzy Zybert. « Glimpses on the History of Idiomaticity Issues », 2004. SKASE, Journal of Theoretical Linguistics, $\mathrm{n}^{\circ}$ 1. www.skase.sk/Volumes/JTL01/kavka.pdf. Consulté le 01 octobre 2021.

Legallois, Dominique. «Les greffes phraséologiques - ou quand la syntaxe se compromet ». Langage, $\mathrm{n}^{\circ}$ 1, vol. 189, 2013, pp. 103-120.

Liontas, John, L. "Teaching Idioms and Idiomatic Expressions across the Second Language Curriculum ». Teaching Essential Units of Language. Beyond single-word Vocabulary, édité par Eli Hinkel. Routledge, Taylor \& Francis, 2019, pp. 55-105.

Lissoni, Antonio (de). Fraseologia italiana o Raccolta di ventimila frasi o modi di dire, per ordine di alfabeto. Milano: Rusconi, 1826.

Makkai, Adam. « The Cognitive Organization of Idiomaticity : Rhyme or Reason? ». Georgetown University Working Papers on Languages and Linguistics, $\mathrm{n}^{\circ}$ 1, 1975, pp. 10-29. 
Mel'čuk, Igor. « Phrasemes in Language and Phraseology in Linguistics ». Idioms : Structural and psychological perspectives, édité par Martin Everaert, Erik-Jan Van der Linden, Andre Schenk et Rob Schreuder. Lawrence Erlbaum, 1995, pp. 167-232.

Morgadinho, Helena. « Les mots du corps dans les locutions et proverbes de la langue française : de leur naissance à leur traduction dans deux autres langues européennes, l'espagnol et le portugais ». Bulletin de Linguistique Appliquée et Générale (BULAG), nº 27, 2002, pp. 182-198.

Pilz, Klaus Dieter. Phaseologie. Redensartenforschung. Metzler, 1981.

Skandera, Paul. Phraseology and Culture in English. De Gruyter, 2007.

Śliwa, Dorota. "Unités polylexicales dans le cadre du syntagme nominal ». Verbum Analecta Neolatina $\mathrm{n}^{\circ}$ 2, vol. 18, 2006, pp. 407-415.

Thun, Harald. Probleme der Phraseologie. Untersuchungen zur wiederholten Rede mit Beispielen aus dem Französischen, Italienischen, Spanischen und Rumänischen. Beihefte zur Zeitschrift für romanische Philologie, vol. 168. De Gruyter, 1978.

TLFi : Trésor de la langue Française informatisé, www.atilf.fr/tlfi. ATILF, CNRS \& Université de Lorraine.

Vinogradov, Viktor V. " Osnovnyye ponyatiya russkoy frazeologii kak lingvisticheskoy distsipliny ». Trudy jubilejnoj naucnoj sessi. Izdatel'stvo Leningradskogo gosudarstvennogo ordena Lenina universiteta, 1946.

Wotjak, Gerd. "Übereinzelsprachliches und Einzelsprachspezifisches bei Phraseolexemen ». Europhras 88. Phraséologie contrastive, édité par Gertrud Gréciano. Université des sciences humaines, 1989, pp. 469-483.

\section{PHRASÉOLOGIE ET IDIOMATOLOGIE : DEUX TERMES CONCURRENTS OU COMPLÉMENTAIRES ?}

$$
\text { Résu mé }
$$

Les discussions terminologiques à propos des différents éléments dont s'occupe la phraséologie (Thun ; Pilz; Gläser) ont été abandonnées depuis des années. Et pourtant, la question est loin d'être résolue au niveau non seulement de la dénomination de ces éléments, mais aussi de celui de leur propre nature. En effet, il continue d'exister une profusion de termes selon les cadres théoriques de tout un chacun (expressions figées, phrasèmes, phraséologismes, unités phraséologiques, etc.). Or, face à cette profusion de dénominations et de typologies, le nom du domaine en question n'a eu qu'un seul concurrent depuis que Bally (vol. I) l'a proposé : celui d'idiomatologie (Guiraud), avec sa variante idiomatik en allemand (Burger et Jaksche), et sa traduction idiomatology en anglais (Makkai ; Kavka et Zybert ; Kavka "Compounding »). Ce constat nous pousse à aller à la recherche d'une explication qui aille au-delà du seul sort aléatoire des mots dans leur consolidation, en retraçant l'histoire de cette concurrence, toute courte qu'elle ait pu être, pour essayer de comprendre les raisons du choix terminologique de Bally et comment ce choix est arrivé à s'imposer. Nous le ferons à travers la comparaison des termes phraséologie et idiotismes et leur coapparition dans deux ouvrages du XIX $\mathrm{X}^{\mathrm{e}}$ siècle (Dupont et Bochet et al.). Cela pourra certainement nous éclairer sur le partage des spécialistes d'aujourd'hui, divisés entre une conception large ou étroite du domaine, avec des typologies plus ou moins inclusives. Mais ce sera surtout l'occasion de réconcilier la phraséologie et l'idiomatologie en tant que domaines complémentaires l'un de l'autre.

Mots clés : Phraséologie; idiomatologie; unités phraséologiques; idiotismes; typologies. 
FRAZEOLOGIA ORAZ IDIOMATYKA:

DWA KONKURENCYJNE CZY UZUPEŁNIAJĄCE SIĘ TERMINY?

\section{Streszczenie}

Dyskusje terminologiczne na temat różnych elementów poruszanych w zakresie frazeologii (Thun; Pilz; Gläser) zostały porzucone na lata. Jednak kwestia ta jest daleka od rozstrzygnięcia, nie tylko jeśli chodzi o nazewnictwo tych elementów, ale także o ich charakter. W istocie nadal istnieje mnogość terminów (wyrażenia stałe, frazemy, frazeologizmy, jednostki frazeologiczne itp.). Jednak w obliczu tej obfitości wyznań i typologii w ramach dyscypliny zwanej frazeologia, nazwa tej dziedziny miała tylko jednego konkurenta, odkąd zaproponował ją Bally (vol. I): idiomatologie (Guiraud) wraz z thumaczeniami na niemiecki - idiomatik (Burger i Jaksche) i angielski - idiomatology (Makkai; Kavka i Zybert; Kavka „Compounding”), w języku polskim - idiomatyka. Ta obserwacja skłania nas do poszukiwania wyjaśnienia tego zagadnienia poprzez odtworzenie historii tej rywalizacji, aby spróbować zrozumieć powody, dla których Bally wybrał daną terminologię i sposób, w jaki ten wybór został narzucony. Dokonaliśmy tego, porównując terminy frazeologia i idiomy oraz ich współwystępowanie w dwóch XIX-wiecznych pracach (Dupont oraz Bochet $i$ in.). To z pewnością rzuca światło na współczesny podział według szerokiej lub wąskiej koncepcji dziedziny, z mniej lub bardziej inkluzywnymi typologiami. Przede wszystkim jest jednak okazją do pogodzenia frazeologii i idiomatyki jako dziedzin uzupełniających się.

Slowa kluczowe: frazeologia; idiomatyka; jednostki frazeologiczne; idiomy; typologie. 